EPJ Web of Conferences 62, 03004 (2013)

DOI: $10.1051 /$ epjconf/20136203004

(C) Owned by the authors, published by EDP Sciences, 2013

\title{
A priori calculations for the rotational stabilisation
}

\author{
Yoritaka Iwata $^{a}$ \\ Graduate School of Science, The University of Tokyo, Hongo 7-3-1, Tokyo 113-0033, Japan
}

\begin{abstract}
The synthesis of chemical elements are mostly realised by low-energy heavyion reactions. Synthesis of exotic and heavy nuclei as well as that of superheavy nuclei is essential not only to find out the origin and the limit of the chemical elements but also to clarify the historical/chemical evolution of our universe. Despite the life time of exotic nuclei is not so long, those indispensable roles in chemical evolution has been pointed out. Here we are interested in examining the rotational stabilisation. In this paper a priori calculation (before microscopic density functional calculations) is carried out for the rotational stabilisation effect in which the balance between the nuclear force, the Coulomb force and the centrifugal force is taken into account.
\end{abstract}

\section{Introduction}

Let low energy heavy-ion collisions be collisions between heavy-ions with incident energies about a few $\mathrm{MeV}$ per nucleon in the centre-of-mass frame. In the theoretical research of low energy heavy-ion collisions, one of the most important purposes is to predict the formation of both new chemical elements and new isotopes, as well as proposing a new method for producing them. Exotic and heavy nuclear synthesis has attracted special attention with a focus on the understanding of nuclei located around the "island of stability". In such a heavy synthesis the competition between long-range Coulomb force and short-range nuclear force is essential. The large Coulomb repulsion tend to increase the break-up of a composite nucleus. Accordingly in order to reduce the Coulomb repulsion, our idea is to introduce the rotational motion to the composite nucleus.

In this paper the role of rotation in nuclear synthesis is examined with respect to the stabilisation of the composite nucleus arising from its rotational motion. A rather new concept for the synthesis of exotic and heavy nuclei utilising three-nucleus simultaneous collisions is reviewed following Ref [1]. Consequently two experimental setups making use of rotational stabilisation are suggested.

\section{Rotational stabilisation}

\subsection{Theoretical modelling}

Here we assume a rotating composite nucleus whose centre-of-mass is fixed to the origin. The mass and proton numbers of a composite nucleus is denoted by $A$ and $Z$. For rotating composite nucleus

\footnotetext{
ae-mail: y.iwata@gsi.de
}

This is an Open Access article distributed under the terms of the Creative Commons Attribution License 2.0, which permits unrestricted use, distribution, and reproduction in any medium, provided the original work is properly cited. 

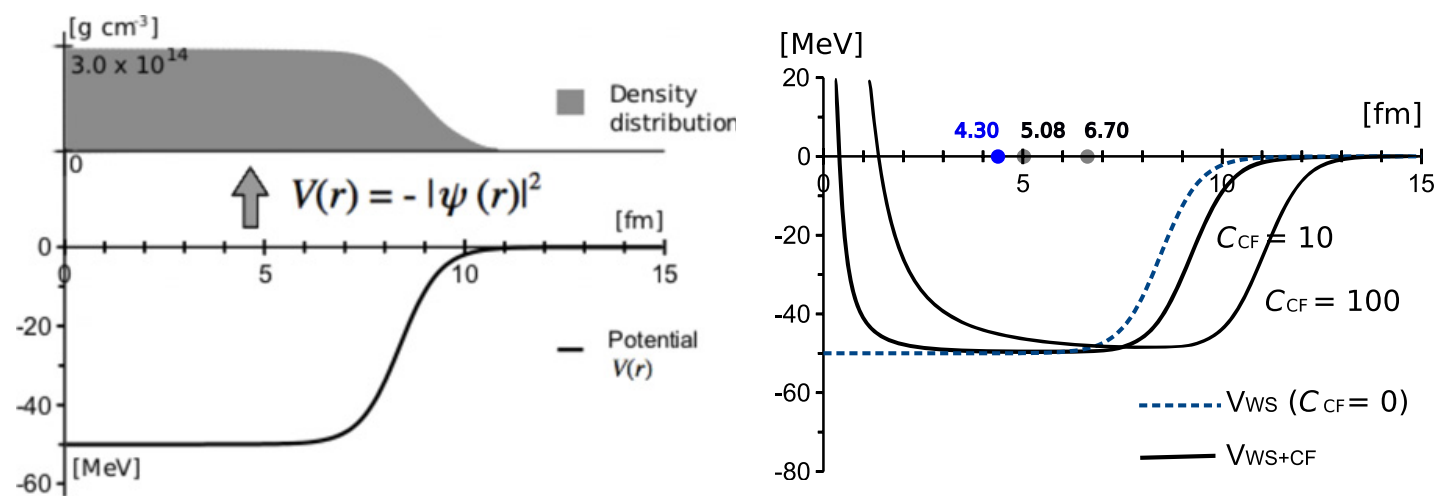

Figure 1. Description of the model. [Left] Process of obtaining the density $|\psi(r)|^{2}$ from the potential $V(r)$ is shown in the original Woods-Saxon potential $(A=350)$, where note that the interaction term represented by $V(r)=-|\psi(r)|^{2}$ is often used in the framework of nonlinear Schrödinger equations. The value $3.0 \times 10^{14}\left[\mathrm{~g} \mathrm{~cm}{ }^{3}\right]$ means the nuclear saturation density. [Right] Centrifugal potentials with different rotation energies are added to the Woods-Saxon potential with $\left(A, C_{\mathrm{CF}}\right)=(350,0)$, where the total density is assumed to be conserved. In addition, the corresponding centre-of-mass is plotted on the horizontal axis: $r=4.30 \mathrm{fm}, 5.08 \mathrm{fm}$, and $6.70 \mathrm{fm}$ for $\left.C_{\mathrm{CF}}\right)=0,10,100$, respectively.

the centrifugal force becomes operational. In order to see the balance between the nuclear force, the Coulomb force and the centrifugal force, a simplified theoretical model calculations is employed (a prior calculation). The nuclear force is assumed to be represented by the Woods-Saxon potential:

$$
V_{\mathrm{WS}}(r)=\frac{V_{0}}{1+\exp \left[\left(r-r_{0} A^{1 / 3}\right) / a\right]},
$$

where $V_{0}=50 \mathrm{MeV}, r_{0}=1.2 \mathrm{fm}$ and $a=0.5 \mathrm{fm}$ are empirical constants. Let the centre-of-mass of the rotating composite nucleus be the origin. The centrifugal-force potential (in the Newtonian mechanics) is represented by

$$
V_{\mathrm{CF}}(r)=\frac{C_{\mathrm{CF}}}{r^{2}}
$$

where $C_{\mathrm{CF}}\left[\mathrm{MeV} \cdot \mathrm{fm}^{2}\right]$ is a parameter associated with the rotation energy and the moment of inertia. Note that $C_{\mathrm{CF}}$ can be controlled by changing the angular momentum (by changing incident energy and impact parameter). Eventually the nuclear-force potential modified by the centrifugal force is represented by

$$
V_{\mathrm{WS}+\mathrm{CF}}(r)=\frac{V_{0}}{1+\exp \left[\left(r-r_{0} A^{1 / 3}\right) / a\right]}+\frac{C_{\mathrm{CF}}}{r^{2}},
$$

where note that the Coulomb potential is represented by $C_{\mathrm{CL}} / r\left(C_{\mathrm{CL}}[\mathrm{MeV} \cdot \mathrm{fm}]\right.$ is a constant). For a model wave function $\psi(r)$ standing for a composite nucleus, we have further assumption:

$$
V_{\mathrm{WS}+\mathrm{CF}}(r)=-\left|\psi_{C_{\mathrm{CF}}}(r)\right|^{2},
$$

is valid if $V_{\mathrm{WS}+\mathrm{CF}}(r)<0$, and $\left|\psi_{C_{\mathrm{CF}}}(r)\right|^{2}=0$ is valid if $V_{\mathrm{WS}+\mathrm{CF}}(r) \geq 0$ (left panel of Fig. 1). The continuum state is intentionally disregarded in this treatment. It is notable that, according to the total density conservation,

$$
\int_{\mathbb{R}}\left|\psi_{C_{\mathrm{CF}}}(r)\right|^{2} d r=\int_{\mathbb{R}}\left|\psi_{C_{0}}(r)\right|^{2} d r
$$



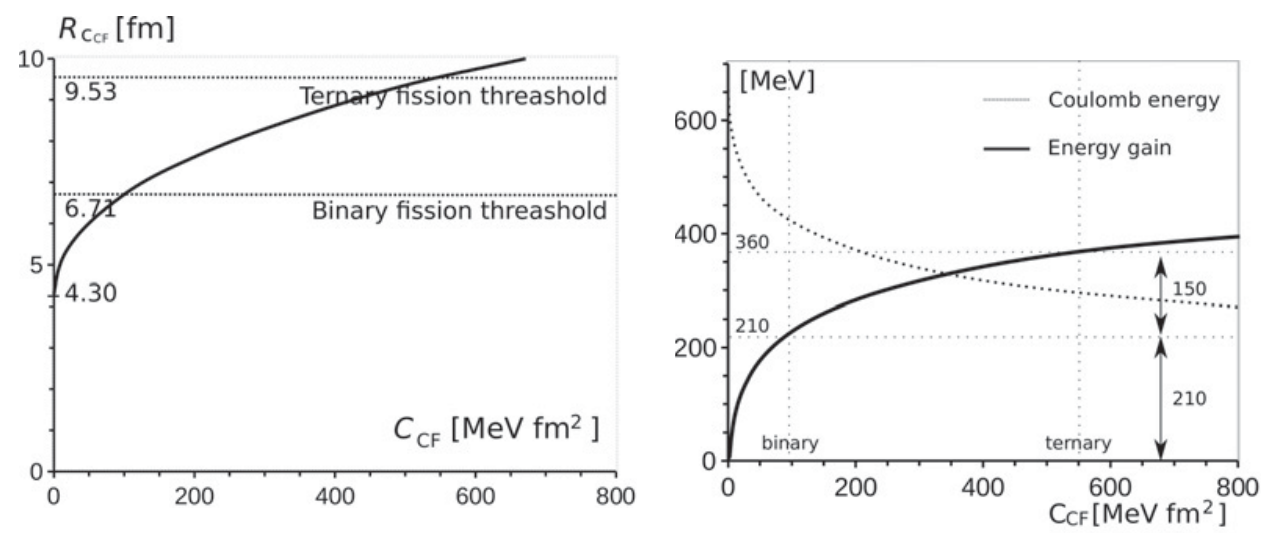

Figure 2. [Left] $C_{\mathrm{CF}}$-dependence of the centre-of-mass for a composite nucleus $(A, Z)=(350,125)$. Threshold $R_{C_{\mathrm{CF}}}$-values (distance between the two half parts) for symmetric binary fission and symmetric ternary fission are also shown. [Right] $C_{\mathrm{CF}}$-dependence of the Coulomb energy (the first term of Eq. (1)) and the Coulomb energy reduction (Eq. (2)). As a result, the composite nucleus elongated to be $R_{C_{\mathrm{CF}}}=6.71 \mathrm{fm}$ and $R_{C_{\mathrm{CF}}}=9.53 \mathrm{fm}$ are more stable compared to the spherical state with $R_{C_{\mathrm{CF}}}=4.30 \mathrm{fm}$ by the reduction $210 \mathrm{MeV}$ and $360 \mathrm{MeV}$, respectively.

must be satisfied regardless of the rotation energy (i.e., regardless of the value of $C_{\mathrm{CF}}$ ). To realise this conservation, a parameter $A$ in $V_{\mathrm{WS}}(r)$ is adjusted case by case. As a result the shape of potential $V_{\mathrm{WS}+\mathrm{CF}}(r)$ changes depending on the rotation energy (right panel of Fig. 1). In this situation, the centreof-mass of composite nucleus (included in a half space), which is defined by

$$
R_{C_{\mathrm{CF}}}=\frac{\int_{0}^{\infty} r\left|\psi_{C_{\mathrm{CF}}}(r)\right|^{2} d r}{\int_{0}^{\infty}\left|\psi_{C_{\mathrm{CF}}}(r)\right|^{2} d r}
$$

moves depending on the rotation energy (left panel of Fig. 2). This value, which is utilised to estimate the elongation of the composite nucleus, indicates the length of a well-balanced stable state. In particular two values are picked out; binary fission threshold $R_{B}=r_{0}(A / 2)^{1 / 3}$, and ternary fission threshold $R_{T}=(13 / 8) r_{0}(A / 3)^{1 / 3}$. Here binary and ternary systems tend to be formed as a composite nucleus in binary and ternary heavy-ion reactions, respectively. Symmetric binary system cannot touch with each other if $R_{C_{\mathrm{CF}}}>R_{B}$, and symmetric ternary system cannot touch with each other if $R_{C_{\mathrm{CF}}}>R_{T}$. In this sense, $R_{B}$ and $R_{T}$ provides the maximal impact parameter for binary and ternary collisions.

In the next step, to examine the elongation effect on the Coulomb energy, the reduction of the Coulomb energy is estimated. If we assume that the centre-of-mass for charge density distribution is the same as the centre-of-mass for the nuclear density distribution, the Coulomb energy is calculated by

$$
E_{\mathrm{Coul}}=\frac{1.44(Z / 2)^{2}}{R_{C_{\mathrm{CF}}}}+E_{\mathrm{int}}
$$

where the first and the second terms of the right hand side mean the binary-type Coulomb energy and the residual Coulomb energy, respectively. In particular the latter term is affected by the nuclear deformation. Using the first term, the Coulomb energy reduction due to the elongation (right panel of Fig. 2) is well approximated by

$$
\triangle E_{\text {Coul }}=1.44(Z / 2)^{2}\left(\frac{1}{R_{0}}-\frac{1}{R_{C_{\mathrm{CF}}}}\right),
$$

where the Coulomb energy reduction arising from the deformation less related with elongation is neglected. 


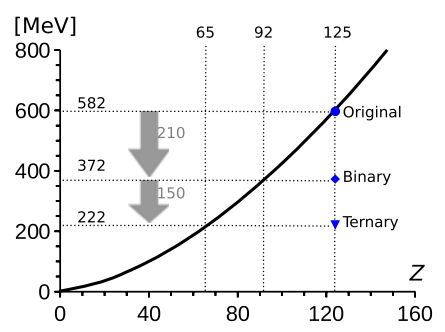

Figure 3. Rotational stabilisation realised by the reduction of Coulomb repulsion. The line shows $E=$ $1.44 Z^{2} /\left(2 r_{0} A^{1 / 3}\right)[\mathrm{MeV}]$. According to Figure 2, the stabilisation energy is found in the comparison.

\subsection{Result: Fusion reaction for superheavy synthesis $(A, Z)=(350,125)$}

In this theoretical model,

1) the elongation distance $R_{C_{\mathrm{CF}}}$ is determined by the rotational energy;

2) the Coulomb energy $E_{\text {Coul }}$ is calculated by the elongation distance;

3 ) the Coulomb energy reduction $\Delta E_{\text {Coul }}$ is obtained for a given rotation energy;

where the distance can be compared with the binary and ternary distances $\left(2 r_{0}(A / 2)^{1 / 3}\right.$ and $(13 / 4) r_{0}(A / 3)^{1 / 3}$, respectively). As shown in the 3 steps, there is only one degree of freedom $\left(C_{\mathrm{CF}}\right)$ in this theoretical treatment among rotation energy (incident energy) and distance (impact parameter).

Let us examine the rotational stabilisation of composite nucleus in a superheavy synthesis. Here we consider the formation of superheavy nucleus $(A, Z)=(350,125)$ in two simplified situations; in almost-symmetric binary fusion

$$
{ }^{175} 63+{ }^{175} 62 \rightarrow{ }^{350} 125
$$

or in almost-symmetric ternary fusion

$$
{ }^{117} 42+{ }^{117} 42+{ }^{116} 41 \rightarrow{ }^{350} 125 .
$$

At the binary and ternary rotation energies (corresponding to $C_{\mathrm{CF}}=100$ and $C_{\mathrm{CF}}=500$ respectively, see left panel of Fig. 2) with maximal impact parameter situations, the corresponding Coulomb energy reduction is 210 and $360 \mathrm{MeV}$ respectively (right panel of Fig. 2). The Coulomb potentials acting on the composite nucleus are similar to that for uranium $(Z=92)$ isotopes in binary case and that for terbium isotopes $(Z=65)$ in ternary case respectively (Fig. 3). As a result the rotational stabilisation effect is high enough to convert instability of superheavy reaction to that of heavy mass or medium mass reactions. Three-nucleus simultaneous collisions are more efficient in which the Coulomb instability of composite nucleus is as much as those of the chemical elements stably existing on earth.

\section{Three-nucleus simultaneous collision}

One method of realising rotating composite nuclei is three-nucleus simultaneous collisions (Configuration 1 of Fig. 4). In such three-nucleus simultaneous collisions the rotational energy is controlled by the projectile energy. Here we define the simultaneous collisions by collisions within $33.3 \times 10^{-22} \mathrm{~s}$, which corresponds to the typical duration of low-energy heavy-ion reactions.

Three-nucleus collisions, which was suggested to be useful to the formation of thin and long structures [1], are expected to be realised in some dense situations in the universe. Two configurations for three-nucleus collisions, which are feasible in experimental facilities on earth, are suggested in Figure 4. Three nucleus simultaneous collisions can be more exactly realised in Configuration 1 than Configuration 2. Indeed, let the projectile be accelerated to $x \%$ of the speed of light, the time interval 


\section{Configuration 1}

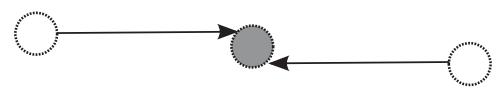

Configuration 2

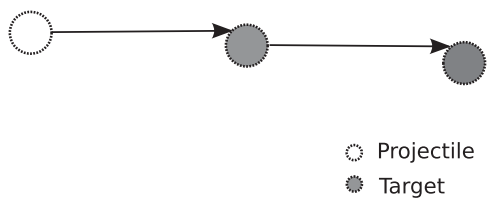

Figure 4. Two configurations of three-nucleus collisions. Two projectile nuclei are designed to collide with target nucleus (at one point) in configuration 1, while one projectile nucleus is designed to experience a sequential collision with two generally different target nuclei (at two different points) in configuration 2.

of the two collisions for Configuration 2 is estimated to be

$$
d /(x \times 0.30) \times 10^{-7} \mathrm{~s} .
$$

where $d \mathrm{~m}$ is the distance between the first and the second targets. For $x=30$ and $d=1$, the time interval is estimated to be $1.1 \times 10^{-8} \mathrm{~s}$, which is far large compared with $33.3 \times 10^{-22} \mathrm{~s}$. Consequently three-nucleus simultaneous collisions are realised only in the case of Configuration 1. On the other hand, the expected cross sections for three-nucleus collisions are much larger in Configuration 2 than Configuration 1.

\section{Conclusion}

A priori calculation has been carried out for the rotational stabilisation effect in which the balance between the nuclear force, the Coulomb force and the centrifugal force is taken into account. The rotational stabilisation has been confirmed to reduce the Coulomb instability quite efficiently.

Three nucleus simultaneous collisions, which was suggested to be useful to the formation of thin and long structures [1], has been confirmed to be useful to the formation of proton-rich or heavy nuclei. Eventually two configuration for realising the three-nucleus collisions are proposed.

This work was supported by HPCI Strategic Programs for Innovative Research (SPIRE) Field 5 "The origin of matter and the universe". The author thanks Prof. J. A. Maruhn for reading the manuscript carefully.

\section{References}

[1] Y. Iwata, K. Iida, and N. Itagaki, Phys. Rev. C 87 (2013) 014609

[2] Y. Iwata, T. Ichikawa, N. Itagaki, J. A. Maruhn, and T. Otsuka, in preparation 